Journal of Theoretical and Applied Mechanics, Sofia, Vol. 47 No. 4 (2017) pp. 37-47

\title{
CALCULATION OF STRESS-DEFORMED CONDITION IN POLYMER NANOCOMPOSITES FILLED WITH MICROCAPSULES WITH LUBRICANT
}

\author{
I.V. Kolesnikov ${ }^{1}$, V.V. Bardushikin ${ }^{2}$, Ph.V. Myasnikov ${ }^{1 *}$ \\ ${ }^{1}$ Rostov State Transport University, Rostov-on-Don, Russia \\ ${ }^{2}$ National Research University of Electronic Technology, Moscow, Russia
}

[Received 09 June 2017. Accepted 4 December 2017]

\begin{abstract}
The article suggests the technology of modifying a polymer matrix by microencapsulation, i. e. the introduction of microparticles (lubricants with nano-additives in polymer shells) into nanocomposites matrix, to form multilevel structures on the tribounit surface. Besides, it suggests the method of predicting the operational elastic properties of multicomponent matrix composites with microcapsules, filled with a liquid substance. The method is based on the generalized singular approximation of the theory of random fields and allows, taking into account the geometric dimensions of the inclusions in the shell. It contains the results of numerical modelling of the effective elastic characteristics (Young's modulus and Poisson's ratio) of composites, based on phenylone with dispersed inclusions (microcapsules), which are glycerin-filled spherical shells of the kapton. The paper investigates the effect of the geometric dimensions of microcapsules and the volumetric content of components on the operational elastic properties of tribocomposites. The developed antifriction nanomaterials with microcapsules are able to create an oriented lubricating coating on the friction surfaces, apply lubrication to a certain friction area and carry out the lubrication portion wise precisely in the necessary contact zone of the bodies.
\end{abstract}

KEY WORDS: Effective moduli of elasticity, matrix composite, inclusion, microcapsule, encapsulation, lubrication, modelling, nanocomposite, tribology

\section{INTRODUCTION}

Increasing the efficiency of friction units of machine parts is traditionally associated with the formation of modified surface layers, or protective coatings, that have special properties. They are the following: low friction coefficient, anisotropy of mechanical properties, increased wear resistance, ability to withstand high specific pressures. The choice of the method of the surface layer modifying is the most complex issue during the development of a technological process, that ensures the achievement of

${ }^{*}$ Corresponding author e-mail: v2k4@mail.ru 
functional properties and resource indices, since only in the final operations of parts processing, such a state of the material surface layer can be attained, that will make it adaptable to operating conditions.

Moreover, the choice of technologies and materials is limited by economic considerations, so the introduction of innovative original nanostructured and nanocomposite materials, advanced technologies and methods of controlling the process of forming a tribocontact surface seems to be promising. The main differences in the behaviour of nanostructured and conventional materials are connected with the fact, that the significant increase of the interface volume fraction plays a considerable role in the first case. It leads to new physical phenomena and unique properties inherent in a nanoscale. A fundamentally new approach is the construction of a polymer matrix by filling it, proceeding from the possibility of implementation of a complex of its new properties, expanding the areas of application.

The researchers suggest the technology of modifying a polymer matrix by microencapsulation, i. e. the introduction of microparticles into the matrix of developed nanocomposites (lubricants with nano-additives) in polymer shells, to form multilevel structures on the tribounit surface [1-3]. The solution of a number of existing technological problems, associated with the uniformity of the distribution of modifiers in the polymer matrix, the growth of the adhesive interaction of the matrix with the metal, while maintaining a predetermined amount of a modifier (microcapsules or oil), that serve as a matrix plasticizer will allow constructing materials with specified operational properties.

Microencapsulation is a qualitatively new direction in the development of science and technology, which is intensively developing at the present time. Ball-shaped capsules are filled with a lubricant, which is liberated only when there are loads on the friction surface. Since the middle of the XX century microcapsules (MC), as substances carriers are widely used all over the world in such fields as chemical industry, medicine, agriculture, etc. The main area of $\mathrm{MC}$ use in mechanical engineering is pressure-processing operations. The use of MC in tribology and tribotechnics has been very limited. The development of microencapsulation technologies in tribotechnics is of great scientific importance, since their introduction has both a significant economic effect and is aimed at preserving the environment from technogenic impact.

The encapsulated substance can be in a solid, liquid or gaseous state. Its content is $7-85 \%$ of the microcapsule mass. The thickness of microcapsule shells is $0.1-10 \mu \mathrm{m}$. They are single- or multilayered, elastic or rigid, depending on the properties of the film-forming agent. The active substance is a lubricant, a corrosion inhibitor, a friction inhibitor, etc. Most often they are encapsulated in the shells of high-molecular compounds of animal and vegetable origin, synthetic polymers and oligomers, paraffins and stearines. Active substances are liberated from microcapsules during the mechanical destruction or dissolution of shells, as well as due to the diffusion. 


\section{MATHEMATICAL MODEL AND CALCULATION RESUlTS}

When developing new materials, using microcapsules, the task is not only to improve the properties of tribocontact, but also to predict the stress-strain condition of these composites. Therefore, this paper considers the problem of numerical modelling of the elastic properties of polymer composites with microcapsules, filled with lubricant.

The methods of theoretical analysis, modelling and calculation of physical and mechanical (in particular, elastic) properties of inhomogeneous materials are based on the prediction of their operational (effective) properties [4-6]. The effective elastic properties of composites are determined by means of a fourth-rank tensor $c^{*}$ ("*", here and further indicates that effective properties are considered), which relates the average values of stresses $\left\langle\sigma_{i j}(\mathbf{r})\right\rangle$ and deformations $\left\langle\varepsilon_{k l}(\mathbf{r})\right\rangle$ in the material:

$$
\left\langle\sigma_{i j}(\mathbf{r})\right\rangle=c_{i j k l}^{*}\left\langle\varepsilon_{k l}(\mathbf{r})\right\rangle, \quad i, j, k, l=1,2,3 .
$$

where $\mathbf{r}$ - the radius vector of the random point of the medium, the angle brackets here and below define ensemble averaging, which for statistically homogeneous composites, i.e. when ergodic hypothesis is fulfilled, coincides with volume averaging [4].

The main problem in calculating the operational elastic properties of inhomogeneous materials with inclusions in liquid or gaseous phases is that, either the shear (fluid) or shear and volume (gas) elastic moduli of such inhomogeneity elements are close to zero. Accordingly, the compliance moduli of such materials tend to infinity [6,7]. In fact, such materials are not simply-connected (especially in porous media).

That is, any such material is not a non-uniform matrix composite, but a homogeneous (matrix material) with developed internal boundaries. Changes in the elastic moduli of such materials will be determined by the geometry of the closed cavities. If we consider this problem broader, then it can be imagined, that gaseous or liquid inclusions are encapsulated in the matrix material. Such approach makes it possible to use the notion of composite inclusion [8]. A detailed survey of the researches devoted to the prediction of the effective elastic properties of inhomogeneous materials with inclusions in liquid or gaseous phases, as well as the problems of modelling and calculation, that arise in this case is given in [9].

It should be noted, that the record of the volume content and the geometry of the inclusions in the liquid or gaseous phase can be carried out within the framework of the generalized singular approximation of the theory of random fields (GSA) [4]. For example, in $[10,11]$, the GSA was used to calculate the effective properties of porous and porous-filled (liquid) inhomogeneous media.

The given paper proposes an approach allowing to combine the calculation of the effective properties of matrix composites with the notion of a combined character 
of the inclusions, with the help of the GSA in the variant of the self-consistency method [4, 5]. This approach, unlike the calculation method considered in [11], allows taking into account the influence of the material and geometric parameters of the microcapsules shells, filled with a liquid on the operational properties of the entire composite. It can provide useful and reliable information about the behaviour of antifriction composite coatings with liquid lubricant inclusions.

To carry out a correct analysis of the effective elastic properties of composites, allowing considering the interaction of heterogeneity, composition, shape, orientation and concentration of components, it is necessary to solve the equilibrium equations for an elastic inhomogeneous medium. However, in general cases it is not possible to obtain a relation for numerical calculations of the effective elastic moduli tensor $c^{*}$. Therefore, various approximations are used for its calculation. One of these approximations, taking into account the above mentioned factors, is GSA [4]. In its framework, only the singular component of Green tensor of the equilibrium equations is used, depending only on Dirac delta function, and a homogeneous body of comparison is introduced, the material constants of which enter the final expression for $c^{*}$ calculation. The physical meaning of GSA is the assumption, that the stress and strains fields are homogeneous within the element of inhomogeneity. In this case, the expression for the tensor $c^{*}$ is the following (the indices are omitted) [4]:

$$
c^{*}=\left\langle c(\mathbf{r})\left(I-g(\mathbf{r}) c^{\prime \prime}(\mathbf{r})\right)^{-1}\right\rangle\left\langle\left(I-g(\mathbf{r}) c^{\prime \prime}(\mathbf{r})\right)^{-1}\right\rangle^{-1},
$$

where $I$ - unit tensor of the fourth rank; $c(\mathbf{r})$ - tensor of elastic moduli; two dashes denote the difference between the corresponding parameters of the inhomogeneous medium and the homogeneous body of comparison, which characteristics are denoted below with the superscript "c": $c^{\prime \prime}(\mathbf{r})=c(\mathbf{r})-c^{\mathbf{c}} ; g(\mathbf{r})$ - integral of the singular component of the second derivative of the Green tensor of the equilibrium equations, which is a fourth-rank tensor. To calculate the components $g_{i j k l}$ of the tensor $g(\mathbf{r})$, at first it is necessary to calculate the components $a_{i k l j}$ of the fourth-rank tensor $A$, and then perform a symmetrization in $a_{i k l j}$ with two pairs of indices $(i, j$ and $k, l)$ [4]. The components $a_{i k l j}$ of $A$ tensor are calculated, using the following relation:

$$
a_{i k l j}=-\frac{1}{4 \pi} \int n_{k} n_{j} t_{i l}^{-1} d \Omega,
$$

where $d \Omega=\sin \theta d \theta d \phi$ - element of the solid angle in a spherical coordinate system, $t_{i l}^{-1}$ - elements of the matrix, inverse to the matrix $\mathrm{T}$ with the elements $t_{i l}=$ $c_{i k l j}^{\mathrm{c}} n_{k} n_{j}, n_{k}$ and $n_{j}(k, j=1,2,3)-$ the components of the vector of the outer normal to the inclusion surface. The components of the normal vector for ellipsoidal 
inclusions with principal semiaxesmare, determined by the relations:

$$
n_{1}=\frac{1}{l_{1}} \sin \theta \cos \phi, \quad n_{2}=\frac{1}{l_{2}} \sin \theta \sin \phi, \quad n_{3}=\frac{1}{l_{3}} \cos \theta .
$$

As it was mentioned, if the ergodicity condition is satisfied, volume averaging (for each composite component) can be used [4], then the entire volume averaging of the material for some random variable $a(\mathbf{r})$ is calculated like that:

$$
\langle a(\mathbf{r})\rangle=\sum_{s} V_{s}\left\langle a_{s}(\mathbf{r})\right\rangle,
$$

where $V_{s}$ and $a_{s}(\mathbf{r})$ - the volume concentration of a component of the $s$-th type and the random variable corresponding to this component, $\sum_{s} V_{s}=1$.

Now, it is necessary to consider the description of the method of predicting the operational elastic properties of multicomponent matrix composites with spherical inclusions in the form of microcapsules, filled with a liquid lubricant. The method is based on GSA, and the calculations themselves are performed in two stages. At the first stage, the tensor of effective moduli of elasticity of a two-component composite simulating spherical microcapsules, filled with a liquid lubricant, is calculated. At the second stage, a "standard" calculation of the effective properties of a multicomponent composite material is performed. Its elements of the inhomogeneity are spherical microcapsules with effective elastic moduli calculated at the first stage, as well as functionalizing additions of other materials in the form of inclusions of different shapes and orientations in the space of the composite and the matrix.

Let us give a more-detailed description of the first stage of the prediction method, which allows taking into account the geometric dimensions of the inclusions in the shell. For this purpose, we consider a two-component matrix composite (the material of the matrix is the material of the microcapsule shells), in which randomly arranged inclusions of the liquid lubricant have the spherical shape of the mean radius $R$. In spite of the fact that the position of the inclusions in the volume of the matrix is random, the material is assumed to be statistically homogeneous. It leads to the presence of an average distance between the inclusions, which may be due to their volume concentration. Indeed, we consider some averaged elementary volume, in which there are three identical inclusions (Fig. 1).

Let the separate inclusion be in the center of the sphere with $R+h_{1}$ radius, where the value $h_{1}$ is such that all three spheres touch each other. Then, the distance between the centers of the spheres is $2\left(R+h_{1}\right)$. Since there are voids in the space between the spheres tangent to each other, it is necessary to choose a value somewhat larger than $h_{1}$ for a characteristic thickness $h$ of the microcapsules shells. We choose 
the following value for $h$ :

$$
h=\frac{h_{1}+h_{2}}{2},
$$

where $h_{2}=\frac{(2-\sqrt{3}) R+2 h_{1}}{\sqrt{3}}$, and value $h_{2}$ corresponds to the case when all three spheres with $R+h_{2}$ radius pass through the center of an equilateral triangle with $2\left(R+h_{1}\right)$ side (Fig. 1).

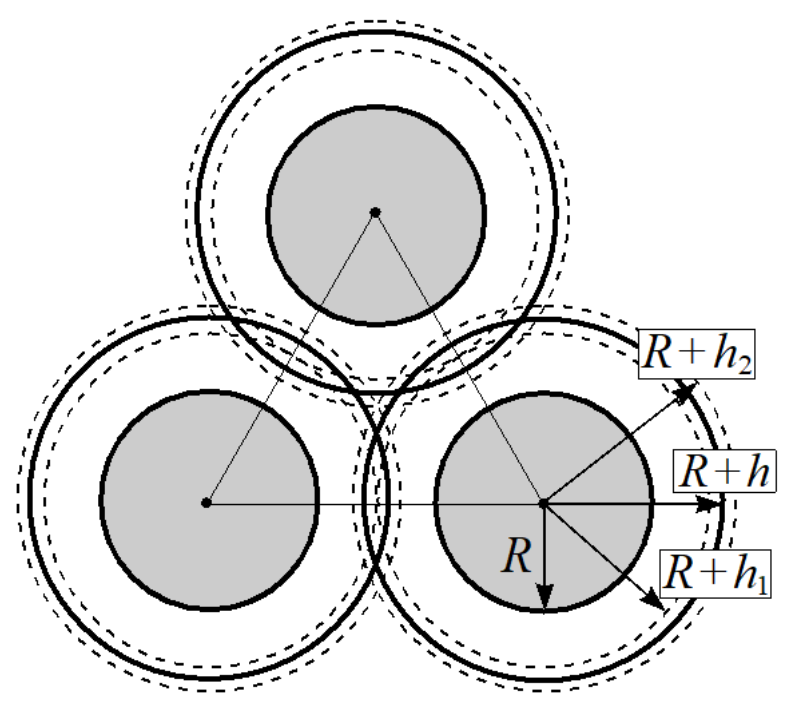

Fig. 1. Geometric model of the first stage of predicting the operational elastic properties of tribocomposites.

For predicting the effective modulus of elasticity of microcapsules, depending on their geometric dimensions, we establish the relationship of these elastic properties with the volume fraction of the "internal" liquid body $V_{\mathrm{b}}$ (or the volume fraction of the shell $\left.V_{\mathrm{sh}}\right)$. Since the characteristic thickness of the shell layer is $h$, and the average radius of the "inner" body is $R$, we have a system of equations, connecting the volume fractions and the geometric dimensions of the components:

$$
V_{\mathrm{b}}+V_{\mathrm{sh}}=1, \quad \frac{V_{\mathrm{sh}}}{V_{\mathrm{b}}}=\frac{\frac{4 \pi(R+h)^{3}}{3}-\frac{4 \pi R^{3}}{3}}{\frac{4 \pi R^{3}}{3}}=\left(1+\frac{h}{R}\right)^{3}-1 .
$$

Hence, the volume fractions $V_{\mathrm{b}}$ and $V_{\mathrm{sh}}$ can be expressed in terms of the dimension- 
less microstructure parameter $h / R$ :

$$
V_{\mathrm{b}}=\left(1+\frac{h}{R}\right)^{-3}, \quad V_{\mathrm{sh}}=1-\left(1+\frac{h}{R}\right)^{-3} .
$$

We carry out model calculations of the operational elastic properties of tribocomposites. We consider a composite with an isotropic matrix of phenylone C2 (Young modulus $E$ at compression is $2822.4 \mathrm{MPa}$, yield stress $\sigma_{\mathrm{y}}$ at compression is 225.9 MPa) [12]. The Poisson's ratio $\nu$ for phenylone can be estimated, using the following equation [13]:

$$
\frac{1-2 \nu}{6(1+\nu)}=\frac{\sigma_{\mathrm{y}}}{E},
$$

from which it follows that $\nu \approx 0.21$.

The phenylone sample is filled with spherical microcapsules. The encapsulated substance is glycerin, the volume modulus of which is $4.4 \mathrm{GPa}$ [14]. Capsule shells are the isotropic aromatic polyimide kapton of DuPont Kapton $® H N$ brand (Young's modulus is $2.5 \mathrm{GPa}$, Poisson's ratio is 0.34) [15].

Given, that the average diameter of the microcapsules, used in the work is $10 \mu \mathrm{m}$, and the volume percentage of glycerin is about $50 \%$, it is possible to calculate the value of the dimensionless structure parameter $h / R$ :

$$
h / R \approx 0.26 \text {. }
$$

Therefore, in subsequent model calculations, the $h / R$ values varied between 0.2 and 0.3 .

The matrix form of the recording was used to carry out model calculations, during operations on tensors [4]. In this case, the nonzero elements $c_{i j}(i, j=1, \ldots, 6)$ of the symmetric matrix of $c$ tensor of the elastic moduli for an isotropic material are expressed in terms of Young's modulus $E$ and Poisson's ratio $\nu$ as follows:

$$
\begin{aligned}
& c_{11}=c_{22}=c_{33}=\frac{E(1-\nu)}{(1+\nu)(1-2 \nu)} ; \\
& c_{44}=c_{55}=c_{66}=\frac{E}{2(1+\nu)} ; \\
& c_{12}=c_{13}=c_{23}=\frac{E \nu}{(1+\nu)(1-2 \nu)} .
\end{aligned}
$$

Prediction of effective modulus of elasticity of microcapsules (the first stage of the method) was carried out with the help of GSA [4]. The most important moment of this approximation is the use of a homogeneous body of comparison, which material 
constants enter the final expression (1) for calculating the effective elastic properties of an inhomogeneous material. One of the widely used approaches for calculating the parameters of a comparison body is the self-consistency method [4,5]. This method was used at the first stage of prediction, when performing the calculations of the effective elastic properties of microcapsules (for every possible value of the microstructure parameter $h / R)$. An iteration procedure was organized to obtain the elastic properties of the comparison body (for each new value $h / R$ ). The values of the elastic modulus tensor (in the matrix form of recording), obtained at the previous iteration step, were taken for its parameters. Characteristics obtained in the Voight approximation $c_{\text {Voight }}=V_{\mathrm{b}} c_{\mathrm{b}}+V_{\mathrm{sh}} c_{\mathrm{sh}}$ [4] were chosen as a zeroth approximation for the parameters of the comparison body.

Such an approach made it possible to perform calculations, despite the fact that the microcapsule "internal" body is a liquid, which volume modulus is different from zero, and the shear modulus is zero. It was assumed that the inclusion of the liquid lubricant had an average radius $R=1$ during the iteration procedure, at each new value $h / R$. In addition, when calculating by the relation (2), the tensor component $g(\mathbf{r})$ it was assumed $l_{1}=l_{2}=l_{3}=R=1$ (for glycerin) and $l_{1}=l_{2}=l_{3}=h / 2$ (for kapton). The exit from the iterative procedure for each $h / R$ was carried out when the maximum difference between the effective moduli was less than 0.01 .

At the second stage of the method, there was conducted the numerical modelling of the operational elastic properties of tribocomposites, based on the phenylone matrix with spherical microcapsules (which effective elastic moduli were calculated at the first stage), using the relation (1). The self-consistency method was used again $[4,5]$ to calculate the parameters of the comparison body. For this purpose, the volumetric content of spherical microcapsules was initially recorded (the geometric dimensions of the "internal" liquid body and shell varied by changing $h / R$ parameter for each value of the microcapsules concentration).

Then, at this microcapsules volume fraction, there was organized an iteration procedure, where the values of the elastic modulus tensor (in the matrix form of recording) obtained at the previous iteration step were taken for the comparison body parameters. Elastic properties in Hill approximation were taken as initial values of the comparison body parameters, i.e. mean arithmetic values, obtained in Reuss $c_{\text {Reuss }}=\left[\sum_{s} V_{s}\left(c_{s}\right)^{-1}\right]^{-1}$ and Voight $c_{\text {Voight }}=\sum_{s} V_{s} c_{s}$ approximations [4]. Calculating the tensor component $g(\mathbf{r})$ by the relation (2), it was assumed that $l_{1}=l_{2}=l_{3}=1$ for all elements of model tribocomposites inhomogeneity. The exit from the iterative procedure was carried out, when the maximum difference between effective moduli was less than 0.01 .

Figure 2 shows the results of numerical calculations of the values of the opera- 

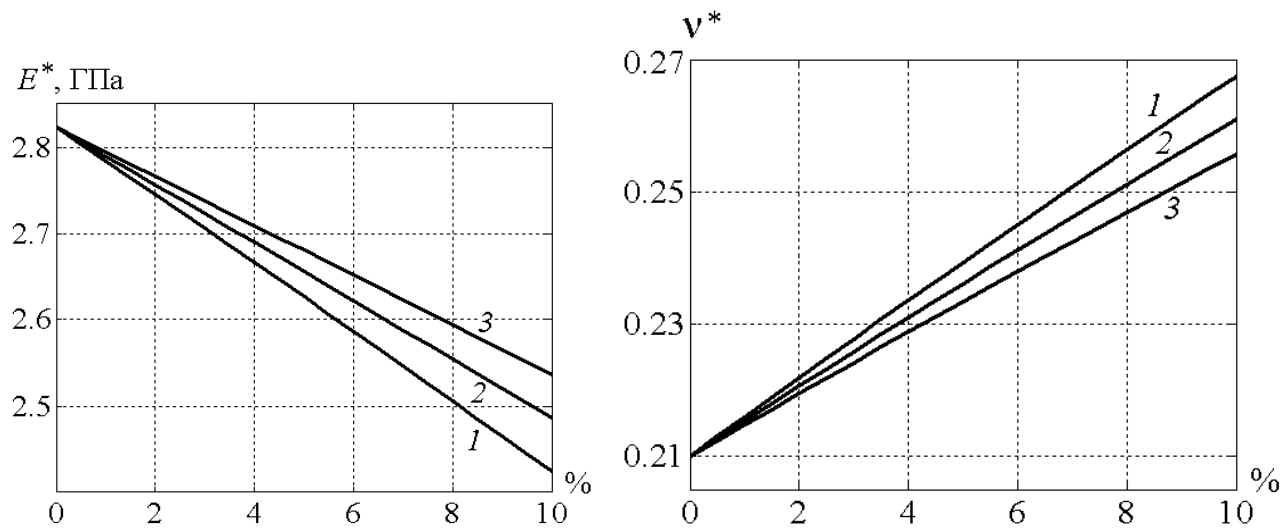

Fig. 2. Operational elastic properties of tribocomposites with a change in the percentage volume content of microcapsules: $\mathbf{1}-h / R=0.2 ; \mathbf{2}-h / R=0.25 ; \mathbf{3}-h / R=0.3$.

tional elastic properties - Young's modulus $E^{*}$ and Poisson's ratio $\nu^{*}-$ of model tribocomposites from the change in the percentage volume content of microcapsules (for three different fixed values of the structural parameter $h / R$ ). The values of $E^{*}$ and $\nu^{*}$ were calculated through the elements $c_{i j}^{*}(i, j=1, \ldots, 6)$ of the tensor $c^{*}$ matrix, according to the following formulas [4]:

$$
E^{*}=\frac{c_{44}^{*}\left(3 c_{12}^{*}+2 c_{44}^{*}\right)}{c_{12}^{*}+c_{44}^{*}}, \quad \nu^{*}=\frac{c_{12}^{*}}{2\left(c_{12}^{*}+c_{44}^{*}\right)} .
$$

The conducted modelling showed that an increase of the percentage volume content of microcapsules leads to an increase of Young's modulus $E^{*}$ and a decrease of Poisson's ratio $\nu^{*}$. In addition, as the values of the structural parameter $h / R$ increase, which is equivalent to an increase of the thickness $h$ of the microcapsules shell (for a fixed value of $R$ ), $E^{*}$ increases and $\nu^{*}$, on the contrary, decreases. We also note, that the dependences $E^{*}$ and $\nu^{*}$ with an increase of the percentage volume content of microcapsules have a character close to linear.

\section{CONCLUSION}

Thus, the main results of the work can be identified as follows:

1. A new method of predicting the operational elastic properties of multicomponent matrix composites with spherical inclusions in the form of microcapsules, filled with a liquid lubricant is considered. The method allows taking into account both the peculiar relative geometric dimensions of microcapsules (by introducing a dimensionless microstructure parameter $h / R$ ) and their concentration in a multicomponent matrix composite. 
2. Using the developed method, there was carried out numerical modelling of the operational elastic properties of a three-component tribocomposite, based on phenolone with spherical microcapsules (encapsulated substance was glycerin, shell was made of kapton) for the given values of the microstructure parameter $h / R$.

The developed antifriction nanomaterials with microcapsules can create an oriented lubricating coating on friction surfaces. It provides better protection of the contacting surfaces from such types of wear as adhesion (due to the passivation of the friction surface by the creation of a protective layer of solid lubricant) and corrosionmechanical (creating a potential barrier for penetration of corrosion-active media into the surface layer). These materials have a low sensitivity to the dependence of physical and chemical properties on the temperature, humidity and dust content of the ambient air. They are able to apply lubrication to a certain friction area, to carry out the lubrication portion wise precisely in the necessary contact zone of the bodies.

\section{ACHNOWLEDGEMENTS:}

The research of Ph.V. Myasnikov and I.V. Kolesnikov supported by the Russian Science Foundation under grant 14-29-00116 was carried out in Rostov State Transport University.

\section{REFERENCES}

[1] Solodovnik, V. D. Microencapsulation, Moscow, Chemistry, 1980. 216 p. (in Russian).

[2] Odintsov, A. V. Capsulation of Mineral Fertilizer Granules in Composite Shells, Thesis of Cand. Tech. Sciences 05.17.08. Ivanovo, 2010, 130 p. (in Russian).

[3] Lopanov, A. N., K. V. Tikhomirova. Physical and Chemical Aspects of Encapsulation Engineering, Belgorod, Belgorod State Technical University Press, 2015, 294 p. (in Russian).

[4] Shermergor, T. D. The Theory of Elasticity of Microinhomogeneous Media, Moscow, Science, 1977, 399 p. (in Russian).

[5] Pankov, A. A. Methods of Self-Consistency of Composites Mechanics, Perm, Perm State Technical University Press, 2008, 253 p. (in Russian).

[6] Vavakin, A. S., R. L. SAlganiK. Effective Elastic Properties of Bodies with Isolated Cracks, Cavities and Rigid Inhomogeneities, Proceedings of the USSR Academy of Sciences, Mechanics of a Solid Body, 1978, No. 2, 95-107 (in Russian).

[7] Levin, V. A., V. A. Lokhin, V. V. Zingerman. On the Construction of Effective Defining Relations for Porous Materials with Randomly Distributed Pores for Finite Deformations and Their Imposition, Proceedings of Universities, The North Caucasus Region, Natural Sciences, 2000, Special Issue,107-115 (in Russian). 
[8] Christensen, R. M. A Critical Evaluation of a Class of Micro-Mechanics Models. J. Mech. Phys. Solids, 38 (1990), 379-404.

[9] Ustinov, K. B. On the Determination of the Effective Elastic Properties of Two-Phase Media. The Case of Isolated Inhomogeneities in the Form of Ellipsoids of Revolution. Advances in Mechanics, (2003), No. 2. 126-168 (in Russian).

[10] BAYUK, I. O. Theoretical Foundations for Determining the Effective Physical Properties of Hydrocarbon Reservoirs. RAO Annual, (2011), No. 12, 107-120 (in Russian).

[11] Bardushinin, V. V., A. I. Sorokin, A. P. Sychev. Modelling of the Operational Elastic Properties of Polymer Composites with Lubricated Spherical Microcapsules and Disperse Inclusions of Alkali-Free Glass. Friction and Lubrication in Machines and Mechanisms, (2015), No. 10, 43-47 (in Russian).

[12] Byrya, A. I., A. I. Sherstyuk, V. N. IVAschenko. Influence of the Content of Ultradispersed Diamonds on the Properties of Composites Based on Aromatic Polyamide. Pore-Depleting and Metal-Working Tools - Techniques and Technology of Its Production and Application, Kiev, Institute of Super Hard Materials named after V. N. BAKUlya, National Academy of Sciences of Ukraine, 2009, No. 12, 336-34 (in Russian).

[13] Kozlov, G. V., D. S. SAnditov. Anharmonic Effects and Physical and Mechanical Properties of Polymers, Novosibirsk, Science, 1994, 261 p. (in Russian).

[14] Physical values. A Reference Book, Ed. I. S. Grigoriev, E. Z. Meilikhov, Moscow, Energoatomizdat, 1991, 1232 p. (in Russian).

[15] http://www.dupont.com/content/dam/dupont/products-and-services/membranes-andfilms/polyimde-films/documents/DEC-Kapton-summary-of-properties.pdf

[16] Gutnikov S. I., B. I. Lazoryak, A. N. Seleznev. Glass Fibers, Moscow, Moscow State University Press, 2010, 53 p. (in Russian). 